\title{
Saccade behaviour in migraine patients
}

\author{
M Cambron ${ }^{1}, \mathrm{~S}$ Anseeuw ${ }^{1}$, K Paemeleire ${ }^{2}$, L Crevits ${ }^{1}$ \\ and Ghent University, Ghent, Belgium \\ Correspondence to: \\ Luc Crevits, $M D, P h D$ \\ Oto-Neuro-Ophthalmology Unit, Department of Neurology \\ Ghent University Hospital \\ De Pintelaan 185 \\ B-9000 Ghent, Belgium. \\ Tel. +3293324529 \\ Fax +3293324971 \\ E-Mailluc.crevits@ugent.be
}

${ }^{1}$ Oto-Neuro-Ophthalmology Unit and ${ }^{2}$ Headache Clinic, Department of Neurology, Ghent University Hospital

\section{Funding: None.}

Contributors: All authors reviewed, edited and approved the final version of the manuscript.

Competing interests: None.

Ethics approval: Ethics approval was obtained from Ethics Committee of the Ghent University Hospital, Ghent, Belgium.

Patient consent: Obtained. 


\begin{abstract}
Background: Voxel-based morphometry studies in migraine patients showed significant grey matter volume reduction in regions involved in the control of saccadic eye movements. We hypothesized these changes would be reflected in dysfunctional saccadic behavior.

Methods: Saccades were recorded by infrared-oculography using 3 different paradigms (progap, pro-overlap and anti-gap). We compared the results of migraine patients $(n=80)$ to those of controls $(n=87)$.

Results: No significant differences were found between migraine patients with $(n=46)$ and without $(n=34)$ aura. Migraine patients showed a saccade behaviour that differed from controls in three respects. In migraine patients, the latencies in the pro-gap paradigm (prosaccade task) were borderline significantly longer. Moreover in both the pro-gap and the prooverlap paradigm we observed a larger intra-individual variation of the latency in migraine patients. However, the biggest difference was that the patients who received migraine prophylactic therapy made significantly more antisaccade errors in the anti-gap paradigm, suggesting that inhibitory saccade control is impaired in migraine patients depending on the severity of the migraine.

Conclusion: We suggest a deficient inhibitory control, reflecting an executive dysfunction in the dorsolateral prefrontal cortex or a dysfunction in the cingulate cortex, is present in migraine patients.
\end{abstract}

Key words: Migraine, eye movement, saccade, oculography, antisaccade. 


\section{Introduction}

Migraine is a common primary headache disorder with widely accepted diagnostic criteria (1). It is characterized by moderate to severe, often unilateral and pulsating, headache attacks that are typically aggravated by physical activity. These attacks can be accompanied by loss of appetite, nausea, vomiting, photo- and phonophobia $(1,2)$. Migraine without aura and migraine with aura are the two main subtypes, with aura features in about $20-30 \%$ of migraine patients (3).

Neuroimaging studies have contributed to the understanding of the neurobiological mechanisms in migraine (4). Studies with voxel-based morphometry (VBM) have been used to study structural changes associated with migraine (5-7). VBM is a fully automated magnetic resonance imaging (MRI) analysis technique that is being increasingly used to detect subtle structural differences in brain tissue composition (5). Compared to controls, migraine patients have significant gray matter volume reductions in, amongst others, the bilateral insula, motor/premotor, prefrontal, cingulate and parietal cortex (5-7). These grey matter volume reductions in migraine patients were correlated with the lifetime headache frequency (4). Several regions which showed significant grey matter volume reduction in migraine, such as the prefrontal and cingulate cortex $(5,6)$, are also involved in the control of saccadic eye movements (8). Furthermore Kim et al. (2009) found a glucose hypometabolism in the bilateral insula, the cingulate, premotor and prefrontal cortex in migraine patients. Correlation analyses showed that regional metabolism of the anterior cingulate cortex had a negative correlation with the lifetime headache frequency (9). We hypothesize that these changes, namely hypometabolism and grey matter volume reduction, may have functional repercussions that can be measured as a dysfunction of saccadic eye movements in migraine. Since Rocca et al. (2006) found no difference in the aforementioned regions involved in saccadic control between migraine patients with or without aura, we did not expect to find differences in the results of saccadic testing between migraine patients with and without aura (7).

There is already functional evidence for involvement of the prefrontal and cingulate cortex in migraine. Several studies concluded that there is a visual attention deficit in migraine patients $(10,11)$. Since the prefrontal and cingulate cortices play a major role in attentional processing, we would expect that migraine patients have difficulties with correctly performing challenging saccadic eye movement tests which require adequate visual attention (12). Infrared-oculography is very suitable to study a broad spectrum of neurophysiological parameters, both of the eye movement and its cerebral control. This allows studies of 
cognitive functions in a qualitative and objective way. To the best of our knowledge, only Wilkinson et al. studied horizontal saccades in migraine patients. They found no significant differences in horizontal saccades in migraine without or with aura compared to headachefree control subjects (13). They used simple tasks where the subjects only had to make reflexive saccades. However, more challenging tasks may be required to elucidate dysfunctions in saccade behaviour. Thus, we focused on more demanding ocular motor tasks using three different saccade paradigms. In the pro-saccade overlap task (further on referred to as pro-overlap), which is a rather simple task, the patient has to make a reflexive saccade towards a stimulus that suddenly appears while the original fixation point stays on (Figure 1A). These reflexive saccades are controlled by the parietal eye fields (PEF) (14). We used this task to compare to the pro-saccade gap task (referred to as pro-gap). The pro-gap task is similar to the pro-overlap task except for a gap in time during which the subject remains without any stimulus, before the target appears (Figure 1B). Turning off the fixation spot before presentation of a new target shortens saccade latencies (the so-called gap-effect), compared to leaving the fixation on (overlap) (15). The gap effect is greatest, reaching 80$100 \mathrm{~ms}$, when the fixation point is removed $200 \mathrm{~ms}$ before the appearance of the target. The shorter latency can be explained by an attentional release that occurs when the subject remains without any stimulus (16). The gap-effect has not been studied so far in migraine patients. Comparing the pro-overlap and the pro-gap task allows us to study that effect which is essentially due to cortical mechanisms and reflects the degree of visual attention. In the anti-saccade gap task (anti-gap), the subject is asked to make an intentional saccade in the direction opposite to the appearing visual target (Figure 1C). We chose the anti-gap task since it requires cognitive processing. The patient has to suppress a reflexive saccadic eye movement in the direction of the stimulus and has to make an intentional saccade towards the opposite direction. This requires much more and more complex visual attention than the former tasks. Several lesion studies have shown that the anti-saccade error percentage is increased in lesions of the dorsolateral prefrontal cortex (DLPFC), while a lesion in the frontal eye fields (FEF) results in an increased latency of intentional saccades $(17,18)$. The number of errors in the anti-saccade task is also increased in lesions that affect the cingulate eye fields (CEF), which is located in the posterior part of the anterior cingulate cortex (ACC). It has been suggested that the CEF has an influence on all frontal regions that play a role in the preparation of saccades, including the DLPFC (18).

In summary, the aim of the present study is to study saccade behavior in migraine patients using three different paradigms, and compare the data to a control population. The hypothesis 
of a dysfunctional saccade behavior in migraine patients was sparked by the description of metabolic and structural changes in the prefrontal and cingulate cortices, both regions known to be involved in the control of saccades.

\section{Methods}

\section{Study population}

Two populations were included: 80 migraine patients and 87 control subjects. A power analysis suggested at least 80 migraine patients and 80 controls should be recruited. Migraine was diagnosed according to the Second Edition of the International Classification of Headache Disorders (ICHD-II) (1) and patients were recruited at the Ghent University Hospital, Department of Neurology. All persons between 18 and 80 years old without disorders that could interfere with the study, were able to participate as controls. The controls were mainly recruited in the environment of the researchers and in the healthy subjects accompanying the patients to a consultation. Exclusion criteria were frequent episodic or chronic tension-type headache and the use of medication known to interfere with saccade control, specifically sedatives and SSRI's (19). However, prophylactic antimigraine drugs were allowed: propranolol $(n=11)$, topiramate $(n=8)$, valproate $(n=7)$, vitamin B2 $(n=3)$, flunarizine $(n=2)$, oxetoron $(n=2)$ and amitriptyline $(n=1)$. The group taking prophylactic medications had $6.06(\mathrm{SD}=4.296)$ migraine days per month. They had on average 10.66 $(\mathrm{SD}=4.985)$ days migraine per month $(\mathrm{n}=34,1$ missing data $)$ before they started prophylactic medication. Migraine patients who did not take any prophylactic medication had on average 4.03 ( $\mathrm{SD}=3.175)$ migraine days a month $(\mathrm{n}=46,1$ missing data) prior to the study. Further on, we will use the term 'headache frequency' to refer to the number of migraine days per month for readability purposes. The headache frequency of the prophylactic group was significantly different from the frequency of the non-prophylactic group at the time of investigation $(\mathrm{P}=0.004)$. Moreover, there were 8 migraine patients who met the ICHD-II criteria for chronic migraine prior to initiation of prophylactic treatment. The patients had migraine for on average 17.52 years $(\mathrm{SD}=13.404)$. The 34 migraine patients on prophylactic medication had migraine for on average 17.12 years $(\mathrm{SD}=13.192)$, while the group who did not take any prophylactic medication had migraine on average for 18.05 years $(S D=14.002)$. Both the migraine patients and the controls were headache-free at the time of the investigation. All migraine patients were headache-free for at least 24 hours before and after the investigation. 


\section{Methods}

Infrared-oculography was used (the ExpressEye system, Freiburg, Germany) for three different tasks: the pro-overlap task, the pro-gap task and the anti-gap task (20).

In the pro-overlap task, the patient was asked to look at the central fixation point (Figure 1A). An extra stimulus ( $4^{\circ}$ to the left or to the right) appeared after $1200 \mathrm{~ms}$. The participant was asked to look at the lateral target as quickly as possible while the central point remained on. The recording of the eye movement stopped $700 \mathrm{~ms}$ after the lateral stimulus appeared. In the gap mode, there was a $200 \mathrm{~ms}$ gap between the extinction of the central fixation point and the appearance of the lateral target. In the pro-gap task, the participant was first asked to look at the central fixation point and then (after the gap), to look at the lateral stimulus as soon as it appeared (Figure 1B). The anti-gap task is similar, except instead of looking at the lateral stimulus, the person was asked to look at the opposite side as quickly as possible (Figure 1C).

\section{Procedure}

The study was approved by the Ethics Committee of Ghent University Hospital (project nr. 2002/258) and each subject signed informed consent. After calibration, the light was dimmed in order to allow the subject to discriminate the stimuli more easily. The participants had to complete the three tasks (pro-overlap, pro-gap and anti-gap), each consisting of 200 trials (100 stimuli to the left and 100 to the right in random order). The sequence of the tasks was randomized for each subject by means of a randomization table made in Excel. Each task was paused after 50 and 100 trials. After 150 trials the subject was allowed to pause anytime he wished to do so after warning the researchers. After each trial there was a pause of $1000 \mathrm{~ms}$ enabling the patient to blink or rest. Before each new task, the subject was given some time to practice in order to assure that the he or she had fully understood the task.

The ExpressEye system registered the eye movements and determined the saccade in each trial. Offline, the interactive software program of the system was used to categorize characteristics of the saccades (20).

\section{Statistical analysis}

For statistical analysis, SPSS version 11.0 for Windows was used (Statistical Package for the Social Sciences, SPSS Inc, Chicago, Il., USA). All variables were checked for impossible values. Each variable was checked for a normal distribution using the Shapiro-Wilk test (normalization test). These tests showed that the data were not normally distributed. For this reason the Mann-Whitney U-test was used to compare the different parameters of migraine 
patients to those of the controls and amongst the migraine patients. This is a non-parametric test to compare two independent samples. We investigated the correlation between two variables using the Spearman correlation coefficient. The significance level was set at P-value $<0.05$.

\section{Measurements}

\section{Parameters}

The parameters determined for the pro-gap and pro-overlap task were: the latencies of the correct saccades with their standard deviation, the number of incorrect saccades, the different types of error and the latency of the correction saccades after a saccade in the wrong direction. The following parameters were determined for the anti-gap task: the latencies of the correct saccades with their standard deviation, the percentage of incorrect saccades, the different types of error, the number of corrections of these saccades and the latency of the correction saccades after a saccade in the wrong direction

'Correct' saccades were defined as saccades in the right direction occurring more than $80 \mathrm{~ms}$ after stimulus presentation.

Four types of errors (incorrect saccades) were determined (Figure 2):

- A-fault: saccades in the wrong direction, not being corrected. In the pro-overlap and pro-gap tasks, one is looking away from the target light instead of looking at the target. In the anti-gap task it means that one is looking to the lateral target instead of looking away from it.

- B-fault: saccades in the wrong direction, being corrected.

- C-fault: stimuli not followed by a saccade ('no reaction').

- D-fault: saccades in the right direction but with a latency of less than 80 ms ('anticipated' saccades, i.e. antisaccades performed before the stimulus was presented or with a latency of 0 $80 \mathrm{~ms})(21)$.

\section{Results}

Age

There was no significant age-difference between the controls and the migraine subjects $(\mathrm{P}>$ $0.05)$; the average age of the controls was 35.3 years $(\mathrm{SD}=13.83)$ (range $16-77)$ and the average age of the migraine patients was 38.3 years $(\mathrm{SD}=11.79)$ (range 15 - 68). 


\section{Gender}

In the control group there were 33 men and 54 women. In the migraine group there were 16 men and 64 women. From recent studies, it is known that there are no significant differences between saccades of men and women (22-24). Therefore, it was not necessary to match for sex differences.

\section{Migraine with aura versus migraine without aura}

In the migraine group there were 42 patients with aura, 34 without aura and 4 patients without exact information about aura. Of the 34 patients with migraine without aura, 16 were not on a prophylactic medication. Of the 42 migraine patients with aura 26 did not take a prophylactic medication. There was no significant difference in any of the saccade parameters studied between migraine with and without aura (data not shown). Thus, we could consider all migraine patients as one group.

\section{Migraine patients versus controls}

Table 1 shows the values of the latencies in the pro-gap and pro-overlap tasks. There was a significant difference in the average latency in the pro-gap task between migraine patients and controls $(\mathrm{P}=0.042)$, and no significant difference in the pro-overlap task. However, the standard deviation of the latencies of the correct saccades significantly differed in migraine patients versus controls in the pro-gap as well as in the pro-overlap tasks (resp. $\mathrm{P}=0.002$ and $P=0.004)$. In both tasks, no significant differences in any type of error were found between migraine and controls.

In the anti-gap task no significant differences between the migraine and the control group were found concerning the latency of the correct anti-saccades (252.4 ms in migraine and $249.2 \mathrm{~ms}$ in controls; $\mathrm{P}=0.302)$ and its standard deviation $(60.0 \mathrm{~ms}$ in migraine and $58.7 \mathrm{~ms}$ in control; $\mathrm{P}=0.608$ ). However we found a significant difference in the percentage saccades in the wrong direction $(\mathrm{P}=0.001)$. The average percentage of saccades in the wrong direction was $24.6 \%$ in the migraine group and $16.0 \%$ in the control group (Table 2). Only the percentage of B-faults (wrongly looking to the target light instead of looking to the opposite side and immediately correcting this error) significantly differed between these two groups (P $=0.001)$. The average percentage of B-faults in the migraine group was $21.1 \%$ and in the control group $12.7 \%$ (Table 2). 
In the migraine group, 34 of the 80 patients used prophylactic anti-migraine drugs (see above). In the pro-gap and pro-overlap tasks, we found no significant differences between the migraine patients with prophylactic medication and those who did not take prophylactic antimigraine drugs. The standard deviation of the latencies of the correct saccades in the pro-gap and the pro-overlap tasks were significantly longer, both in the migraine patients with and without prophylactic medication versus the control subjects (respectively $\mathrm{P}=0.002$ and $\mathrm{P}=$ 0.038 for the pro-gap and $\mathrm{P}=0.005$ and $\mathrm{P}=0.048$ for the pro-overlap task). The latency of the correct saccades in the pro-gap paradigm did not remain significant when subgroups of migraine patients with $(\mathrm{P}=0.079)$ and without $(\mathrm{P}=0.116)$ prophylaxis were compared to controls.

In the anti-gap task we found one significant difference between patients with or without prophylactic drugs: migraine patients who take prophylactic medication made significantly more saccades in the wrong direction than those without daily medication $(\mathrm{P}=0.029)$. Migraine patients without prophylactic medication only borderline significantly differed $(\mathrm{P}=$ 0.056) from the control subjects concerning the amount of saccades made in the wrong direction (Figure 3). Concerning the percentage of saccades in the wrong direction, there was no significant difference between the different groups of prophylactic medication and the nonprophylactic group $(\mathrm{P}=0.118)$.

\section{Correlation with headache frequency}

We investigated the relationship between the headache frequency (i.e. number of migraine days per month) and the test performance. In migraine patients on prophylaxis, the headache frequency prior to initiation of treatment was used to adequately reflect the severity of the migraine disorder in these patients. For the anti-gap task we found that the headache frequency was positively correlated with the percentage saccades in the wrong direction $(\mathrm{P}=$ 0.001) (Figure 4). There was a negative correlation between the latency of the wrong saccades and the headache frequency $(\mathrm{P}=0.042)$ (Figure 5). In the pro-gap and pro-overlap tasks no correlation was found between the headache frequency and the performance on these tests (P $>0.05)$.

\section{DISCUSSION}

Since studies using VBM in migraine patients have shown significant grey matter volume reduction in the prefrontal and the cingulate cortices (5-7), both regions that are involved in 
the control of saccadic eye movements (8), we hypothesized this would be reflected in a dysfunctional saccade behavior.

As none of the saccade parameters differed significantly between migraine patients with aura or without aura, we could consider the migraine patients as one group. We did not exclude patients who took prophylactic medication as the sample might otherwise reflect a milder end of the migraine spectrum. As could be expected, the group who took prophylactic medication had a higher number of migraine days per months prior to initiation of prophylaxis than those who did not take any prophylactic medication. The pharmacological treatment effects on saccadic eye movements have been studied previously (19). The following prophylactic antimigraine drugs were used by our patients: propranolol $(n=11)$, topiramate $(n=8)$, valproate $(\mathrm{n}=7)$, vitamin B2 $(\mathrm{n}=3)$, flunarizine $(\mathrm{n}=2)$, oxetoron $(\mathrm{n}=2)$ and amitriptyline $(\mathrm{n}=1)$.

No effect has been found of tricyclic antidepressants on latency or on error rate of saccadic eye movements (25). Sawaguchi investigated the effect of propranolol when applied on the prefrontal cortex (26) and found no effect on the performance of an ocular motor delayedresponse task. Flunarizine has no effect on the latency of saccadic eye movements but the effect on the error rate was not investigated (27). We found no studies on the effect of riboflavin, topiramate, valproate and oxetoron on saccadic eye movements.

\section{Pro-gap and pro-overlap}

Compared to controls, the migraine patients showed a larger standard deviation of the latencies in the pro-overlap as well as in the pro-gap tasks suggesting a higher degree of variability making a reflexive (automatic) saccade to a visual target. In controls, reflexive saccades and anti-saccades show a small intrapersonal variation of the latency (28). Large variations of intra-individual results in ocular motor tests are commonly ascribed to various exogenous (diseases, drugs) and endogenous (motivation, learning, fatigue, habituation, circadian rhythm) factors (29). In our study, a larger variation of latency was only found in the pro-overlap en pro-gap tasks of migraine patients. In terms of ocular motor circuitry, the parietal-collicular path is common to these pro-saccades, while anti-saccades have their major input from the prefrontal cortex. Thus, an instable parietal-collicular input, perhaps reflecting changes in visual attention, in migraine is hypothesized.

The latencies of the pro-overlap task saccades were similar in migraine patients and controls, while those of the pro-gap task saccades were borderline larger in migraine. We can explain these findings by a smaller gap effect in migraine patients than in controls. The gap-effect can 
be conceptualized as the result of an attentional release or a fixation desinhibition when the subject remains without a stimulus (30). Thus a cortical deficit could explain a reduced gap effect (11). This finding did not remain significant when we compared the migraine subgroups with and without prophylactic medication separately to the control group, and can thus not be attributed to one of both subgroups.

\section{Anti-gap}

Anti-saccades are intentional saccades made in the direction opposite to an appearing target. Several studies have shown that the anti-saccade error rate (i.e. saccades to the target) is increased in lesions of the DLPFC, while a lesion in the FEF results in an increased latency of the correct saccades $(17,18)$. It has been suggested that the number of errors in the antisaccade task is also increased in lesions that affect the CEF $(14,18)$. The CEF seems to have an influence on all frontal regions that play a role in the preparation of saccades, including the DLPFC (18).

The major finding of the present study was the significantly increased number of anti-saccade errors in migraine patients, reflecting a deficient saccade behavior to inhibit reflexively glancing at the target. The patients proved to understand the task very well as the errors were corrected (type B error). Furthermore, the number of uncorrected anti-saccades (type A error) was not significantly increased in migraine patients. As the latency of the anti-saccades was not significantly longer in migraine patients than in controls, a dysfunction of the circuitry involving the DLPFC rather than the FEF is suggested. An increase of anti-saccade errors is in accordance with neuropsychological studies that have provided evidence for cognitive, especially executive dysfunctions in migraine patients, both during acute attacks as well as interictally $(31,32)$. As both the cingulate and the prefrontal cortex play a major role in different attentional processing systems (12), an attentional deficit might explain why the number of type B errors was increased.

However, an increased anti-saccade rate was only found in the subgroup of migraine patients on prophylactic medication, although the subgroup with no prophylactic medication was only borderline significantly different. A pharmacologic effect cannot be excluded, but is not supported by the available literature data, at least for propranolol, tricyclic antidepressants and flunarizine (25-27). However it should be stated that the effect of a prophylactic migraine treatment on the anti-saccades has never been investigated.

We rather suggest that the severity of the migraine disorder was the determining factor, as the group of patients receiving prophylactic medication had a higher frequency of migraine 
headache days (before prophylaxis) and therefore took drugs. This hypothesis is supported by the finding that the percentage saccades in the wrong direction was positively correlated with the headache frequency. This suggests that the more severe the migraine disorder, the more migraine patients have difficulties to suppress reflexive saccades and thus make more mistakes. We also found a negative correlation between the latency of the wrong saccades and the headache frequency, suggesting a release of reflexive saccades. One could imagine that if the suppression of these reflexive saccades decreases due to a problem in the DLPFC, they would be more easily evoked which explains a reduction of the latency as the deficit gets worse.

Our findings suggest an inhibitory dysfunction in saccade circuits involving the prefrontal or the cingulate cortex, especially in patients with frequent migraine attacks. This is in accordance with previous findings of neuroimaging studies that showed a significant reduction of grey matter volume in regions involved in the control of saccades, which was correlated with the headache frequency $(33,6)$. This supports our hypothesis that the abnormalities found in migraine patients are more evident as the patient has more attacks. Furthermore more disturbances in memory, attention and visuomotor speed processing were found in migraineurs experiencing higher frequency of attacks in previous cognitive (11).

In conclusion, we found abnormal saccade behaviour in migraine patients, especially of the anti-saccades. We hypothesize that grey matter volume reduction in the prefrontal and cingulate cortex accounts for this abnormal saccade behaviour. An attentional deficit might underly the smaller gap effect found in the migraine group. However, this has to be confirmed in future studies.

The extent of the anti-saccade deficit seems to depend on the number of migraine headache days the patient has suffered. Although the clinical significance of the anti-saccade disturbance in migraine remains rather speculative, our study underlines once more that the behavioural consequences of the migraine disorder stretch beyond episodic symptoms. 


\begin{tabular}{|c|c|c|c|c|c|c|c|c|c|c|c|c|}
\hline & \multirow{2}{*}{\multicolumn{5}{|c|}{ MIGRAINE }} & \multirow{2}{*}{\multicolumn{5}{|c|}{ CONTROL }} & \\
\hline & & & & & & & & & & & & \multirow[b]{2}{*}{ P-VALUES } \\
\hline & & $\begin{array}{r}\text { Mean } \\
(\mathrm{ms})\end{array}$ & SD & $\begin{array}{c}\text { Median } \\
(\mathrm{ms})\end{array}$ & Min & Max & $\begin{array}{r}\text { Mean } \\
(\mathrm{ms})\end{array}$ & SD & $\begin{array}{c}\text { Median } \\
(\mathrm{ms})\end{array}$ & Min & Max & \\
\hline \multirow[t]{2}{*}{ Pro-gap } & $\begin{array}{c}\text { Average latency } \\
\text { of correct } \\
\text { saccades }\end{array}$ & 183.2 & 37.34 & 178.5 & 115 & 305 & 169.9 & 27.60 & 163.0 & 120 & 272 & 0.042 \\
\hline & SD of latency & 45.8 & 21.29 & 40.00 & 19 & 123 & 36.7 & 18.12 & 31.0 & 16 & 113 & 0.002 \\
\hline \multirow[t]{2}{*}{$\begin{array}{c}\text { Pro- } \\
\text { overlap }\end{array}$} & $\begin{array}{c}\text { Average latency } \\
\text { of correct } \\
\text { saccades }\end{array}$ & 211.6 & 31.92 & 205.0 & 149 & 281 & 206.9 & 30.79 & 204.00 & 142 & 286 & 0.475 \\
\hline & SD & 50.6 & 18.02 & 49.0 & 22 & 103 & 42.7 & 15.88 & 38.5 & 18 & 90 & 0.004 \\
\hline
\end{tabular}

Table 1: Pro-gap and pro-overlap: Average latencies and standard deviation. Average latencies (in milliseconds) (the mean, median, SD, minimum and maximum of the mean latency scores of each patient) and standard deviation (SD) of these latencies (the mean, median, SD, minimum and maximum of the individual SD) in the pro-gap and prooverlap tasks in the migraine and the control group. Statistical significance between the two groups: values in bold are significant $(\mathrm{P}<0.05)$. 


\begin{tabular}{|c|c|c|c|c|c|c|c|c|c|c|c|}
\cline { 2 - 13 } \multicolumn{1}{c|}{} & \multicolumn{4}{|c|}{ MIGRAINE } & \multicolumn{5}{c|}{ CONTROL } & P-VALUES \\
\hline Anti-gap & Mean (\%) & SD & Median (\%) & Min & Max & Mean (\%) & SD & Median (\%) & Min & Max & \\
\hline $\begin{array}{c}\text { Percentage saccades in } \\
\text { the wrong direction }\end{array}$ & 24.6 & 18.94 & 17.5 & 3 & 87 & 16.0 & 14.78 & 12.0 & 3 & 79 & $\mathbf{0 . 0 0 1}$ \\
\hline Percentage A-faults & 3.5 & 5.05 & 1.5 & 0 & 26 & 3.3 & 5.26 & 1.1 & 0 & 27 & 0.235 \\
\hline Percentage B-faults & 21.1 & 18.11 & 15.8 & 2 & 85 & 12.7 & 11.20 & 9.4 & 1 & 60 & $\mathbf{0 . 0 0 1}$ \\
\hline Percentage C-faults & 0.8 & 1.37 & 0.5 & 0 & 8 & 1.0 & 2.26 & 0.0 & 0 & 14 & 0.622 \\
\hline Percentage D-faults & 0.9 & 1.64 & 0.0 & 0 & 9 & 0.6 & 0.96 & 0.0 & 0 & 4 & 0.351 \\
\hline
\end{tabular}

Table 2: Anti_gap: Average values for the percentage of the errors. Average values for the percentage of errors in the anti-gap task in the migraine and the control group. Values in bold are significant $(\mathrm{P}<0.05)$. 


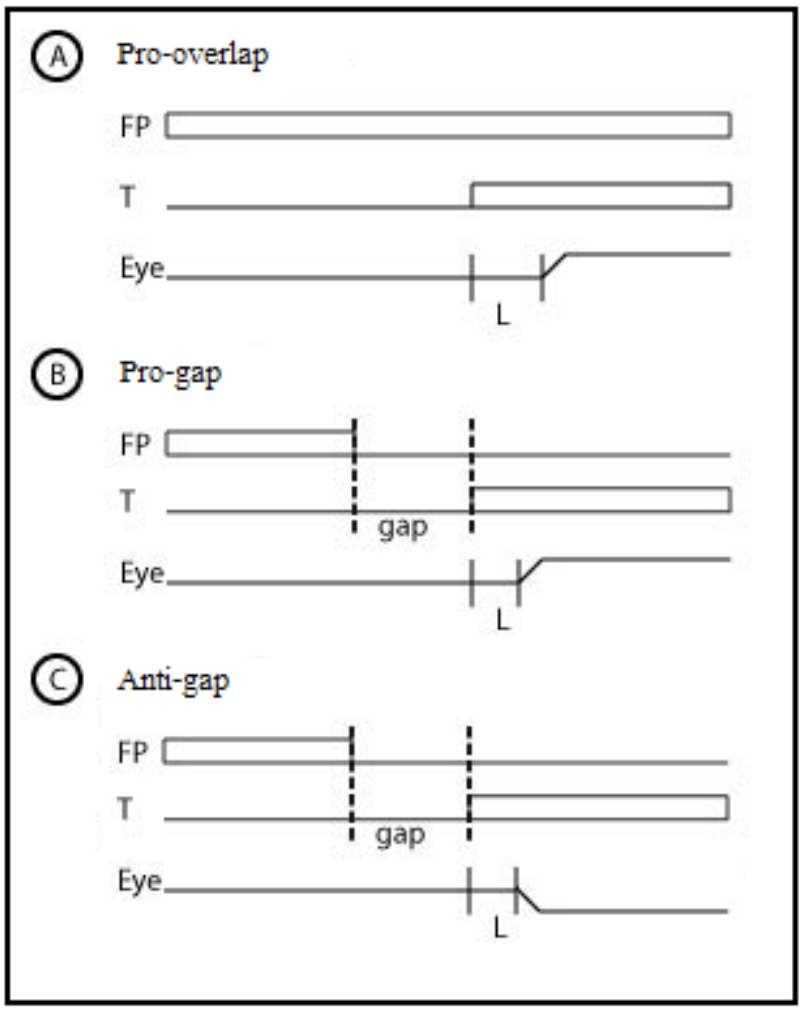

Figure 1: Illustration of the different tasks. A. Pro-overlap: The subject is asked to look at the central fixation point (FP). An extra stimulus (T) appears after $1200 \mathrm{~ms}$. The participant is asked to look at the $\mathrm{T}$ as quickly as possible while the FP remains on. B. Pro-gap: This task is similar to the pro-overlap task; the only difference is that the FP disappears after $1000 \mathrm{~ms}$ and the subject remains $200 \mathrm{~ms}$ without any stimulus. Then the T appears and the subject is asked to look at it as quickly as possible. C. Anti-gap: Similar to the pro-gap task, the FP disappears after $1000 \mathrm{~ms}$ and $200 \mathrm{~ms}$ later the T appear. In this task the subject has to look to the opposite site of the appearing $\mathrm{T}$ instead of looking to the $\mathrm{T}$ itself. (FP: 'fixation point' or central fixation point, T: 'target' or extra stimulus, Eye: eye movement, L: latency) 


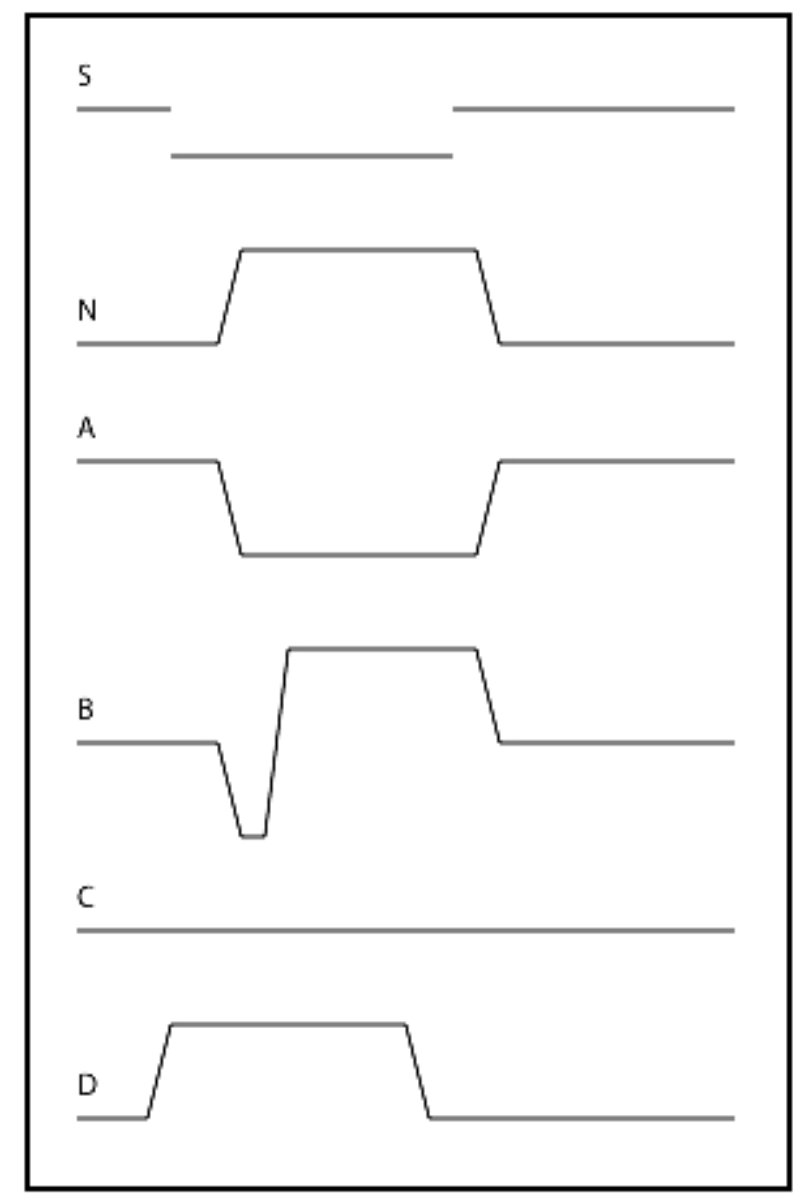

Figure 2: Illustration of the possible faults made in the anti-saccade task. S: Stimulus in the anti-saccade task. The deviation of the stimulus curve downwards represents a stimulus appearing at the right (down: right, up: left).

$\mathrm{N}$ : Correct anti-saccade. In the anti-saccade task the subject has to look to the opposite site of the appearing target. A: A-fault, saccade made in the wrong direction and not corrected. B: B-fault, saccade made in the wrong direction and corrected. C: C-fault, no reaction on the stimulus. D: D-fault, saccades made too early. This saccade cannot be the response of the stimulus. 


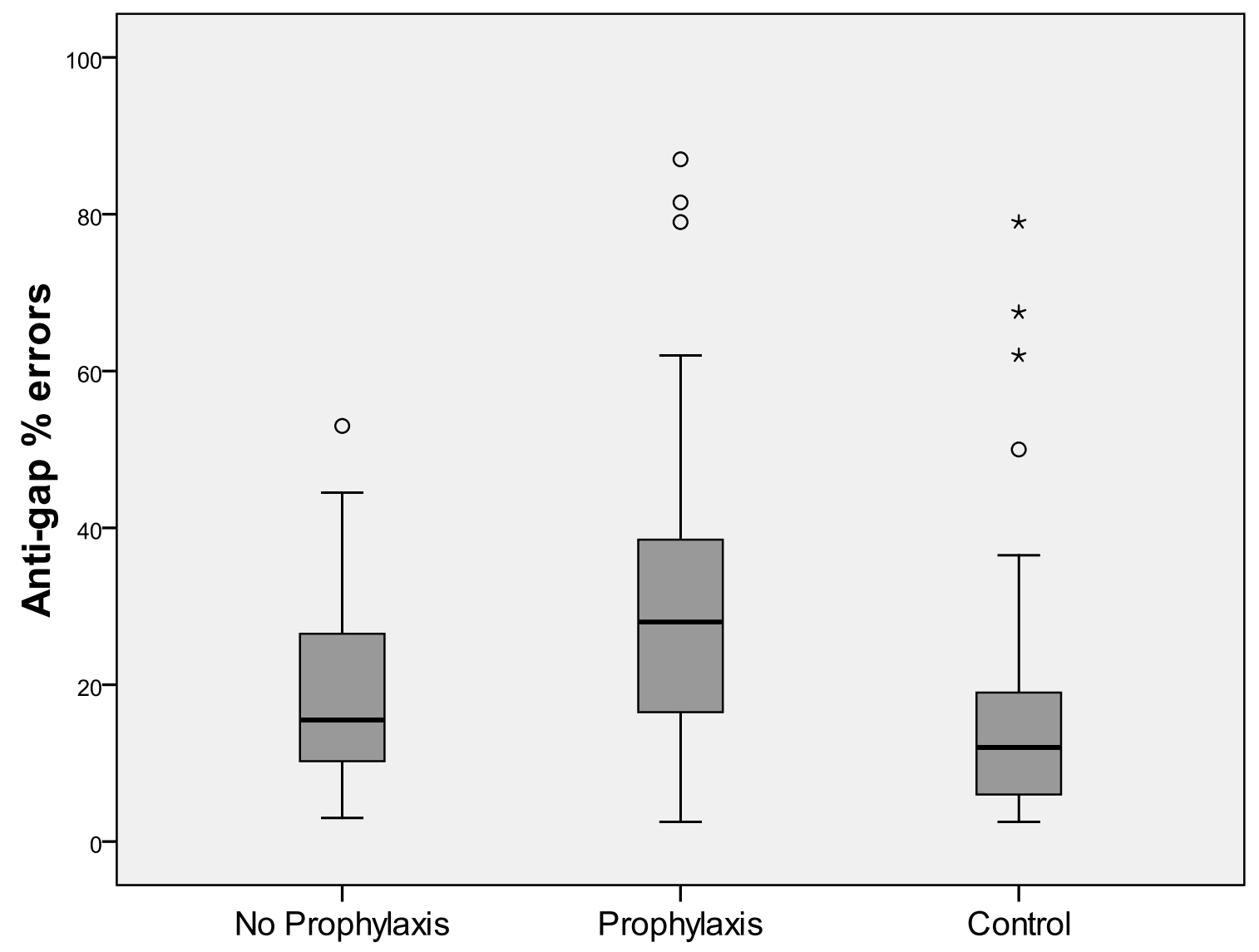

Figure 3: A comparison of the percentage anti-gap errors between different groups using a boxplot. The different boxplots represent the control group $(n=87)$, the migraine groups with prophylaxis $(n=34)$ and without prophylaxis $(\mathrm{n}=46)$. The ${ }^{\circ}$ and $*$ are outliers. 


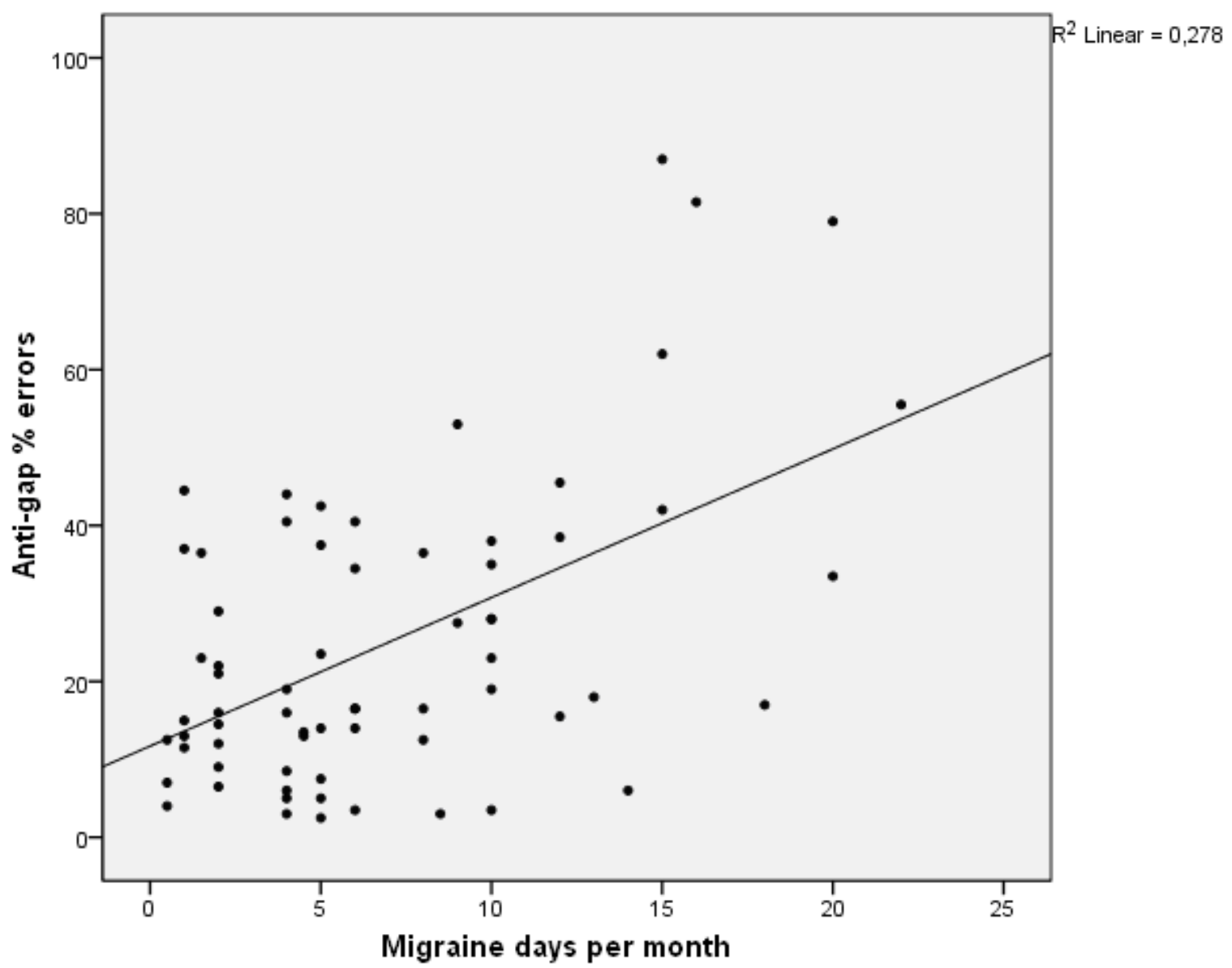

Figure 4: Scatterplot of the correlation between the migraine days per month and the percentage errors in the anti-gap task. This figure gives a graphical representation of the positive correlation between the migraine days per month and the percentage errors in the anti-gap task $(\mathrm{P}=0.001)$. 


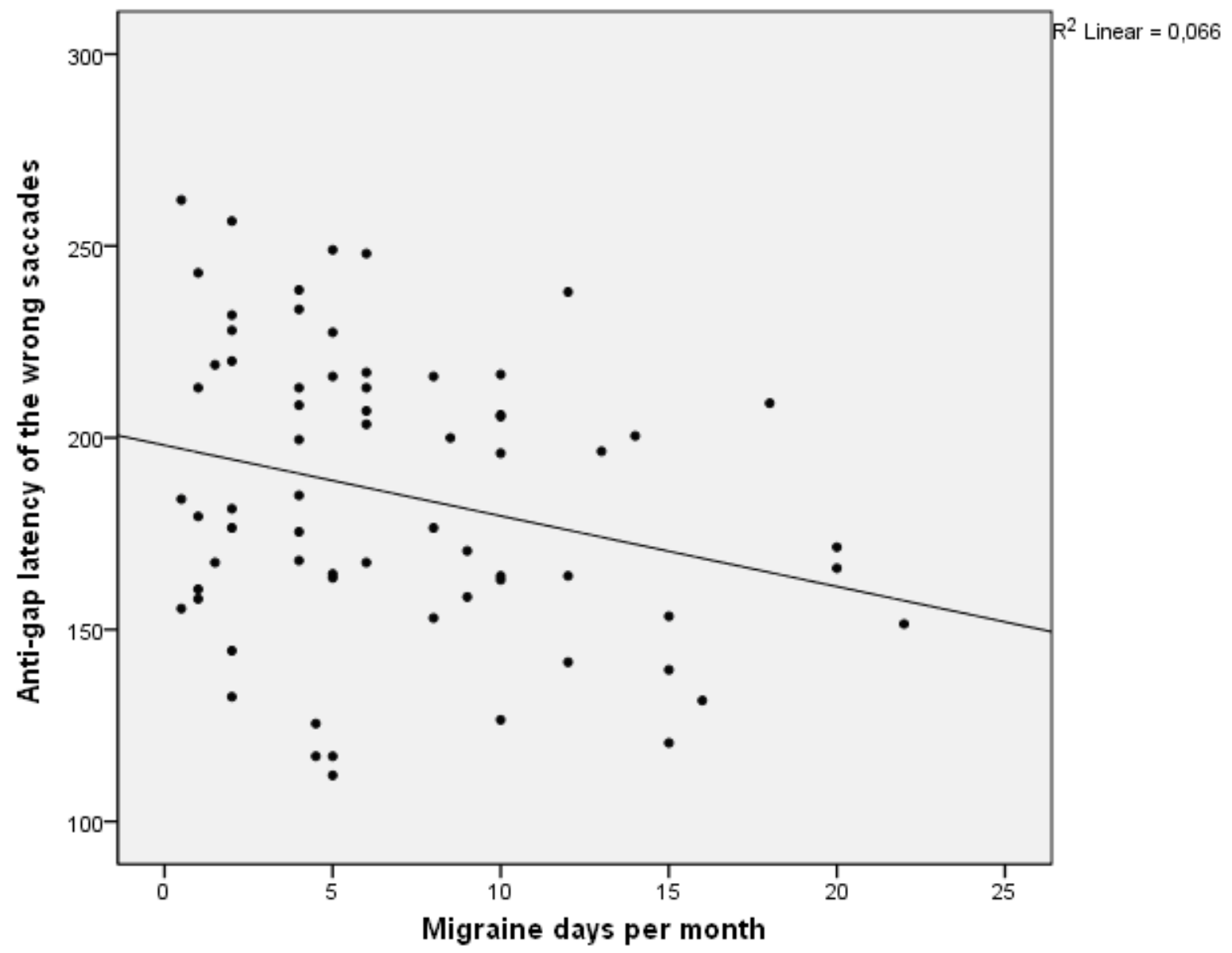

Figure 5: Scatterplot of the correlation between the migraine days per month and the latency of the wrong saccades. This figure gives a graphical representation of the correlation between the latency of the wrong saccades and the migraine days per month $(\mathrm{P}=0.042)$. 


\section{REFERENCES}

1. Headache Classification Committee of the International Headache Society. The International Classification of Headache Disorders. Cephalalgia 2004; 24:24-43.

2. Goadsby PJ, Lipton RB, Ferrari MD. Migraine--current understanding and treatment. N Engl J Med. 2002; 346(4):257-70.

3. Manzoni GC, Torelli P. Migraine with and without aura: a single entity? Neurol Sci. 2008 May;29 Suppl 1:S40-3

4. May A. A review of diagnostic and functional imaging in headache. J Headache Pain. 2006; 7(4):174-84.

5. Kim JH, Suh SI, Seol HY, Oh K, Seo WK, Yu SW, et al. Regional grey matter changes in patients with migraine: a voxel-based morphometry study. Cephalalgia. 2008; 28(6):598-604.

6. Valfrè W, Rainero I, Bergui M, Pinessi I. Voxel-based morphometry reveals gray matter abnormalities in migraine. Headache 2008; 48:109-17.

7. Rocca MA, Ceccarelli A, Falini A, Colombo B, Tortorella P, Bernasconi L, et al. Brain gray matter changes in migraine patients with T2-visible lesions: a 3-T MRI study. Stroke 2006; 37(7):1765-70.

8. Pierrot-Deseilligny Ch, Müri RM, Nyffeler T, et al. The role of the human dorsolateral prefrontal cortex in ocular motor behavior. Ann N Y Acad Sci 2005; 1039:239-51.

9. Kim JH, Kim S, Suh S-I, Koh S-B, Park K-W and Oh K. Interictal metabolic changes in episodic migraine: A voxel-based FDG-PET study. Cephalalgia 2009; 30(1) 53-61

10. Villa TR, Correa Moutran AR, Sobirai Diaz LA, et al. Visual attention in children with migraine: a controlled comparative study. Cephalalgia 2009; 29(6):631-4.

11. Calandre EP, Bembibre J, Arnedo ML, et al. Cognitive disturbances and regional cerebral blood flow abnormalities in migraine patients: their relationship with the clinical manifestations of the illness. Cephalalgia 2002; 22(4): 291-302.

12. Ng CW, Noblejas MI, Rodefer JS, et al. Double dissociation of attentional resources: prefrontal versus cingulate cortices. J Neurosci. 2007; 27:12123-31.

13. Wilkinson F, Karanovic O, Ross EC, et al. Ocular motor measures in migraine with and without aura. Cephalalgia 2006; 26:660-71.

14. Gaymard B, Pierrot-deseilligny. Neurology of saccades and smooth pursuit. Curr Opin Neurol. 1999; 12 (1):13-19

15. Vernet M, Yang Q, Gruselle M, Trams M, Kapoula Z. Switching between gap and overlap pro-saccades: cost or benefit? Exp Brain Res. 2009; 197(1):49-58

16. Jin Z, Reeves A. Attentional release in the saccadic gap effect. Vision Res. 2009; 49(16):2045-55.

17. Pierrot-Deseilligny C, Rivaud S, Gaymard B, et al. Cortical control of reflexive visually-guided saccades. Brain 1991; 114:1473-85.

18. Pierrot-Deseilligny C, Müri RM, Nyffeler T, et al. The role of the human dorsolateral prefrontal cortex in ocular motor behavior. Ann N Y Acad Sci 2005; 1039:239-51.

19. Reilly JL, Lencer R, Bishop JR, et al. Pharmacological treatment effects on eye movement control. Brain Cogn. 2008; 68:415-35. Review.

20. Hartnegg K, Fischer B. A turn-key transportable eye-tracking instrument for clinical assessment. Behav Res Methods Instrum Comput. 2002; 34:625-9

21. Munoz DP, Dorris MC, Paré M, et al. On your mark, get set: brainstem circuitry underlying saccadic initiation. Can J Physiol Pharmacol. 2000; 78:934-44.

22. Fujiwara K, Kunita K, Toyama H.Changes in saccadic reaction time while maintaining neck flexion in men and women. Eur J Appl Physiol. 2000; 81(4):317-24. 
23. Nyberg S, Wahlström G, Bäckström T, Poromaa IS. No difference in responsiveness to a low dose of alcohol between healthy women and men. Pharmacol Biochem Behav. 2004; 78(3):603-10.

24. Wilson SJ, Glue P, Ball D, Nutt DJ. Saccadic eye movement parameters in normal subjects. Electroencephalogr Clin Neurophysiol. 1993; 86(1):69-74.

25. Wilson SJ, Bailey JE, Alford C, et al. Effects of 5 weeks of administration of fluoxetine and dothiepin in normal volunteers on sleep, daytime sedation, psychomotor performance and mood. Psychopharmacol. 2002; 16(4):321-31.

26. Sawaguchi T. Attenuation of delay-period activity of monkey prefrontal neurons by an alpha2-adrenergic antagonist during an oculomotor delayed-response task. J Neurophysiol. 1998; 80(4):2200-5.

27. Casucci G, Di Costanzo A, Riva R, et al. Central action of cinnarizine and flunarizine: a saccadic eye movement study. Clin Neuropharmacol. 1994; 17(5):417-22.

28. Crevits L, De Clerck M, Van Maele G. Reliability of saccades. Neuro-Ophthalmology. 2000;24:319-25.

29. Schalen L, Pyykko I, Juhola M, Magnusson M, Jantti V, Henriksson N. Intraindividual variation in oculomotor performance in man. Acta Otolaryngol Suppl. 1984;406:212-7.

30. Crevits L, Vandierendonck A. Gap effect in reflexive and intentional prosaccades. Neuropsychobiology 2005; 51:39-44.

31. Farmer K, Cady R, Bleiberg J, et al. Sumatriptan nasal spray and cognitive function during migraine: results of an open-label study. Headache 2001; 41:377-84.

32. Mulder EJ, Linssen WH, Passchier J, et al. Interictal and postictal cognitive changes in migraine. Cephalalgia 1999; 19:557-65.

33. Paemeleire K. Brain lesions and cerebral functional impairment in migraine patients. $\mathbf{J}$ Neurol Sci. 2009; 282:134-1369. 